
\title{
25 Research Soure \\ Maintenance phototherapy for the treatment of early stage mycosis fungoides
}

\section{Soumaya Gara}

Universite de Tunis El Manar Faculte de Medecine de Tunis

Noureddine Litaiem

Universite de Tunis El Manar Faculte de Medecine de Tunis

\section{Takwa Bacha}

Universite de Tunis El Manar Faculte de Medecine de Tunis

Djamila Sandra OUEDRAOGO ( $\square$ ouedraogo.djamila.sandra@gmail.com )

Centre Hospitalier Universitaire Yalgado Ouedraogo

\section{Yosra Jmour}

Universite de Tunis El Manar Faculte de Medecine de Tunis

\section{Faten Zeglaoui}

Universite de Tunis El Manar Faculte de Medecine de Tunis

\section{Research article}

Keywords: phototherapy, mycosis fungoides, PUVA therapy, narrowband UVB therapy

Posted Date: September 10th, 2019

DOI: https://doi.org/10.21203/rs.2.14186/v1

License: (c) (i) This work is licensed under a Creative Commons Attribution 4.0 International License.

Read Full License 


\section{Abstract}

Background : Phototherapy has been a mainstay of treatment of early stages (la-lla) of mycosis fungoides (MF). Despite this, there is no internationally standardized phototherapy regimen schedule for

MF. Efficacy of maintenance therapy is poorly evaluated especially in patients with dark phototype (IV-VI). Methods : Thirty patients with early stage MF treated with PUVA therapy and narrowband UVB therapy from January 2004 to January 2016 at a single institution were retrospectively reviewed. Recurrence rate and recurrence-free survival were assessed in patients who received maintenance phase and in those who underwent follow-up. Results : Seventeen patients had patch stage disease while 16 patients had plaque stage disease. Most of the patients $(22,73 \%)$ had dark phototype. Nineteen patients received NBUVB therapy, while 11 patients received PUVA. Mean follow-up period was $36,1+13$ months. There was no significant association between the recurrence rate and recurrence free survival in patients who received maintenance phase and those who underwent follow-up. Conclusions: Phototherapy is a safe and effective treatment option for patients with early stage MF. Evidence supporting the use of maintenance phase for the treatment of early stages MF is lacking.

\section{Background}

Mycosis fungoides (MF) is the most common type of cutaneous T cell lymphoma accounting for about $50 \%$ of all primary cutaneous lymphomas (1). The diagnosis is based on clinical, histological and immunophenotypical findings.

The term MF is usually restricted to the classic "Alibert-Bazin" type characterized by the typical progression of patches, plaques, and tumors. Early stages of MF (stages IA-IIA) are characterized by limited or generalized patches and/or plaques with no significant lymph node involvement.

Phototherapy has been a mainstay of treatment of MF for the past 50 years and is recommended as firstline treatment of early-stage MF according to the most recent European organization for Research and Treatment of Cancer (EORTC) consensus (2). Guidelines for phototherapy of MF have been proposed by the United States Cutaneous Lymphoma Consortium (USCLC) (3).

Despite this, there is no internationally standardized phototherapy regimen schedule for MF. Questions regarding the efficacy of maintenance phase are still debated (4).

Studies comparing recurrence rates in patients who received maintenance phase and those who underwent follow-up are scarce including a relatively small proportion of patients with dark phototype (phototypes IV-VI) (5-8).

The present study aimed at assessing the efficacy of phototherapy in Tunisian patients with MF treated with phototherapy and evaluate the efficacy of maintenance phase.

\section{Methods}


We retrospectively reviewed all patients treated with phototherapy for MF in a 12-year period (from January 2004 to January 2016). We included in the study subjects diagnosed with early-stage MF (stage la - Ila) who underwent a whole-body phototherapy course: psoralen and ultraviolet A (PUVA) therapy (wavelengths between 320 and $400 \mathrm{~nm}$ ) or narrow-band ultra-violet B (NB-UVB) therapy (wavelengths between 311 and $313 \mathrm{~nm}$ ), in the department of dermatology, Charles Nicolle hospital, Tunis, Tunisia. Pathological and immunohistochemical diagnostic confirmation was made in all included cases.

The evaluation of each patient included a thorough physical examination, a complete blood count and serum chemistries. CT scans of the chest and abdomen were performed if clinically indicated. Patients were classified according to the TNM staging proposed by the International Society of Cutaneous Lymphoma/European Organization of Research and Treatment of Cancer in 2007 (9).

Treatment protocol:

The ultraviolet light-based therapy was delivered by a Waldmann cabin $7001 \mathrm{~K}$ UVA-UVB (Herbert Waldmann $\mathrm{GmbH}$ and Co. KG, Deutschland). Patients treated with PUVA therapy had received 8methoxypsoralen two hours before each session. The psoralen's dose was calculated according to body weight $(0,6 \mathrm{mg} / \mathrm{kg})$. Phototherapy sessions were carried out three times per week. Both PUVA and NB-UVB therapy starting doses were established according to the patient's phototype. Dosage increments (20\% every two sessions) were established if the previous session did not cause erythema. The regimen was sustained until complete clinical clearance (induction phase), then continued for 4 weeks (consolidation phase). Maintenance treatment was proposed at the end of the consolidation phase once weekly for 2 weeks, followed by once every 2 weeks for 8 weeks and then once every 4 weeks.

Treatment response was established as follow: complete response as clearing of $100 \%$ of lesions, partial response as clearing between $50 \%$ and $100 \%$ of lesions, and therapeutic failure: clearing less than $50 \%$ of lesions or progressing disease.

Remission was defined by a maintained complete response for at least 4 weeks. Recurrences were defined by a relapse of the disease after achieving remission.

Statistical analysis:

Data for all cases were compiled electronically and analyzed using SPSS v 19 (SPSS Inc., Chicago, IL, USA). For quantitative variables, the results were expressed as mean and standard deviation. Otherwise, the results were expressed as frequencies for qualitative variables. $T$-test and Chi-square were respectively used for comparison of continuous or parametric variables (Mann-Whitney and Fisher exact test when appropriated). Recurrence-free survival was assessed by the Kaplan-Meier method with logrank test. Significance level of 0.5 was used.

\section{Results}


Thirty patients were enrolled. The main features of our patients are summarized in Table 1. Seventeen patients had patch stage disease while 16 patients had plaque stage disease. Most of the patients (22, 73\%) had dark phototype (Fitzpatrick phototype IV-VI).

There was no significant difference between the recurrence rates in patients with dark phototype vs light phototype ( $p>0.05)$. Recurrences were significantly more frequent in plaque stage MF treated with NBUVB ( $3 / 5$ vs $2 / 14 ; p=0.043)$, while there was no significant difference of recurrence rates in patients treated with PUVA ( $2 / 7$ vs $1 / 4 ; p=0.71)$. There was no significant association between the recurrence rates and the following parameters: age, gender (men $8 / 23$, women $2 / 7 ; p=0.475$ ), and maintenance phototherapy ( $3 / 8$ vs $5 / 22 ; p=0.64)$. There was no significant difference between recurrence-free survival in patients who received maintenance therapy and those who underwent follow-up in both PUVA (26 vs 33 months, $p=0.63$ ) and NB-UVB (21 vs 48 months, $p=0.3)$ subgroups.

\section{Discussion}

This retrospective study, which included mostly patients with dark phototype, confirmed the efficacy and safety of phototherapy in the treatment of MF. Recurrence rates and recurrence-free survival were not significantly different in patients who received maintenance phase and those who underwent follow-up.

The treatment of MF with PUVA therapy was first described by Gilschrest et al in 1976 (10). UVA penetrates the entire dermis and possibly the subcutaneous tissue. Therefore, PUVA represents a good therapeutic option for plaque stage disease (up to $85 \%$ of patients with stage IA experienced complete response) $(4,11,12)$. Complete response rates were slightly lower in patients treated with NB-UVB $(54 \%$ to $90 \%$ ) (4). Therefore, NB-UVB is considered a convenient therapeutic option for patch stage disease. The efficacy of NB-UVB is however partial in folliculotropic and plaque-type MF. This could be explained by its limited dermal and adnexal penetration.

Treatment strategy depends also on skin phototype. Pavlosky et al found that patients with skin phototypes I-III reached higher complete remission rates and required a lower dose to achieve complete remission compared to patients with dark phototype (13). In the largest series to date of MF patients treated with phototherapy, only 6 patients with dark phototype received NB-UVB (8). In our study, nineteen patients received NB-UVB therapy. Response rates were comparable with previous reports and thus despite including an important proportion of patients with dark phototype $(14,15)$.

The benefits of maintenance PUVA therapy is still contested. To date, only rare single-center studies evaluated recurrence rates and recurrence-free survival in patients who received maintenance therapy (5- 
7). None of these studies has shown a significant difference in both recurrence rates and recurrence-free survival (5-7).

Maintenance NB-UVB is also still debated. Encouraging results were reported in a few studies $(16,17)$. These results should, however, be interpreted with caution due to distinct maintenance regimens.

There are several concerns regarding maintenance phototherapy. As discussed earlier, evidence of its efficacy is lacking. In the other, phototherapy is associated with an increased incidence of carcinomas. Non-melanoma skin cancers are related to phototherapy in a dose-dependent manner (18). Therefore, maintenance therapy could increase the risk of the development of skin cancers without gaining substantial therapeutic outcomes.

Limitations of this study are the relatively small number of enrolled patients, its retrospective and monocentric nature which may lead to missing data and selection bias. Maintenance treatment was proposed to patients at the end of the consolidation phase. We found no significant association between the recurrence rates in patients who received maintenance therapy and those who underwent follow-up. These results may be confounded by an indication as maintenance therapy could be encouraged for patients with more extensive or severe disease.

\section{Conclusions}

Phototherapy is a safe and effective treatment option for patients with early stage MF, regardless of patient's phototype. Treatment regimen should include induction and consolidation phases. The aim of the latter is to treat subclinical lymphoma. Evidence supporting the use of maintenance phase for the treatment of early stages MF is lacking.

\section{References}

1. Swerdlow SH, Campo E, Pileri SA, Harris NL, Stein H, Siebert R, et al. The 2016 revision of the World Health Organization classification of lymphoid neoplasms. Blood. 2016;127(20):2375-90.

2. Trautinger F, Eder J, Assaf C, Bagot M, Cozzio A, Dummer R, et al. European Organisation for Research and Treatment of Cancer consensus recommendations for the treatment of mycosis fungoides/Sézary syndrome - Update 2017. Eur J Cancer. 2017;77:57-74.

3. Olsen EA, Hodak E, Anderson T, Carter JB, Henderson M, Cooper K, et al. Guidelines for phototherapy of mycosis fungoides and Sézary syndrome: A consensus statement of the United States Cutaneous Lymphoma Consortium. J Am Acad Dermatol. 2016;74(1):27-58.

4. Grandi V, Fava P, Rupoli S, Alberti Violetti S, Canafoglia L, Quaglino P, et al. Standardization of regimens in Narrowband UVB and PUVA in early stage mycosis fungoides: position paper from the 
Italian Task Force for Cutaneous Lymphomas. J Eur Acad Dermatol Venereol JEADV. 2018;32(5):683-91.

5. Sánchez MA, González T, Gaitán MF, Zuluaga A, Jiménez SB, de Galvis YT. Is PUVA maintenance therapy necessary in patients with early-stage mycosis fungoides? Evaluation of a treatment guideline over a 28-month follow-up. Int J Dermatol. 2011;50(9):1086-93.

6. Hernández Z, Peñate Y, Hernández-Machín B, Pérez-Méndez L, Suárez-Hernández J, Hernández J, et al. Treatment of stage la and lb mycosis fungoides with psoralen UVA monotherapy: an observational study in tertiary hospitals in the Canary Islands. Int J Dermatol. 2014;53(11):1417-22.

7. Wackernagel A, Hofer A, Legat F, Kerl H, Wolf P. Efficacy of 8-methoxypsoralen vs. 5-methoxypsoralen plus ultraviolet $\mathrm{A}$ therapy in patients with mycosis fungoides. $\mathrm{Br} \mathrm{J}$ Dermatol. mars 2006;154(3):519-23.

8. Nikolaou V, Sachlas A, Papadavid E, Economidi A, Karambidou K, Marinos L, et al. Phototherapy as a first line treatment for early stage mycosis fungoides: The results of a large retrospective analysis. Photodermatol Photoimmunol Photomed. 2018 Sep;34(5):307-313. doi: 10.1111/phpp.12383.

9. Olsen E, Vonderheid E, Pimpinelli N, Willemze R, Kim Y, Knobler R, et al. Revisions to the staging and classification of mycosis fungoides and Sezary syndrome: a proposal of the International Society for Cutaneous Lymphomas (ISCL) and the cutaneous lymphoma task force of the European Organization of Research and Treatment of Cancer (EORTC). Blood. 2007;110(6):1713-22.

10. Gilchrest BA, Parrish JA, Tanenbaum L, Haynes HA, Fitzpatrick TB. Oral methoxsalen photochemotherapy of mycosis fungoides. Cancer. 1976;38(2):683-9.

11. Adışen E, Tektaş V, Erduran F, Erdem Ö, Gürer MA. Ultraviolet A1 Phototherapy in the Treatment of Early Mycosis Fungoides. Dermatology. 2017;233(2-3):192-8.

12. Olek-Hrab K, Silny W, Dańczak-Pazdrowska A, Osmola-Mańkowska A, Sadowska PA, Polańska A, et al. Ultraviolet A1 phototherapy for mycosis fungoides: UVA1 A1 phototherapy for MF. Clin Exp Dermatol. 2013;38(2):126-30.

13. Pavlotsky F, Barzilai A, Kasem R, Shpiro D, Trau H. UVB in the management of early stage mycosis fungoides. J Eur Acad Dermatol Venereol. 2006;20(5):565-72.

14. Almohideb M, Walsh S, Walsh S, Shear N, Alhusayen R. Bath Psoralen-ultraviolet A and Narrowband Ultraviolet B Phototherapy as Initial Therapy for Early-stage Mycosis Fungoides: A Retrospective Cohort of 267 Cases at the University of Toronto. Clin Lymphoma Myeloma Leuk. 2017;17(9):604-12.

15. Elcin G, Duman N, Karahan S, Ersoy-Evans S, Erkin G, Karaduman A, et al. Long-term follow-up of early mycosis fungoides patients treated with narrowband ultraviolet B phototherapy. J Dermatol Treat. 2014;25(3):268-73.

16. Querfeld C, Rosen ST, Kuzel TM, Kirby KA, Roenigk HH, Prinz BM, et al. Long-term follow-up of patients with early-stage cutaneous T-cell lymphoma who achieved complete remission with psoralen plus UV-A monotherapy. Arch Dermatol. 2005;141(3):305-11.

17. Roenigk HH, Kuzel TM, Skoutelis AP, Springer E, Yu G, Caro W, et al. Photochemotherapy alone or combined with interferon alpha-2a in the treatment of cutaneous T-cell lymphoma. J Invest Dermatol. 
1990;95(6 Suppl):198S-205S.

18. McGregor JM, Crook T, Fraser-Andrews EA, Rozycka M, Crossland S, Brooks L, et al. Spectrum of p53 gene mutations suggests a possible role for ultraviolet radiation in the pathogenesis of advanced cutaneous lymphomas. J Invest Dermatol. mars 1999;112(3):317-21.

\section{Abbreviations}

MF: mycosis fungoides

PUVA: psoralen and ultraviolet A

NB-UVB: narrow-band ultraviolet B

EORTC: European organization for Research and Treatment of Cancer

USCLC: United States Cutaneous Lymphoma Consortium

\section{Declarations}

\section{Ethics approval and consent to participate:}

This was a retrospective study. Ethical principals were respected in accordance with the Declaration of Helsinki. All personal data were kept anonymous. An approval by the institutional ethics committee is deemed unnecessary according to national Tunisian regulations where the study was conducted (legislation: http://www.dpm.tn/images/pdf/cc01012015fr.pdf)

\section{Consent for publication}

Not applicable

\section{Availability of data and materials}

The datasets used and/or analyzed during the current study are available from the corresponding author on reasonable request.

Competing interests

The authors declare that they have no competing interests

\section{Funding:}

No funding was obtained for this study.

\section{Authors' contributions}


NL designed the study. FZ wrote the protocol. TB and DSO managed the literature searches and analyses. YJ undertook the statistical analysis, and SG, NL and DSO drafted of the manuscript. All authors contributed to and have approved the final manuscript.

\section{$\underline{\text { Acknowledgements }}$}

Not applicable.

\section{Tables}

Table 1: Clinical characteristics of patients diagnosed with MF and treated with phototherapy.

\begin{tabular}{|l|l|l|}
\hline \multicolumn{2}{|l|}{ Age (years) } & $56,6 \pm 15,6[14-80]$ \\
\hline \multicolumn{2}{|l|}{ Sex (male/female) } & $23 / 7$ \\
\hline \multicolumn{2}{|l|}{ Diagnosis delay (months) } & $46 \pm 52,8[2-180]$ \\
\hline \multirow{2}{*}{ Phototype } & II & 1 \\
\cline { 2 - 3 } & III & 5 \\
\cline { 2 - 3 } & IV & 16 \\
\cline { 2 - 3 } & V & 8 \\
\hline Clinical aspects of skin lesions & Patch & 17 \\
\cline { 2 - 3 } & Plaque & 16 \\
\hline Pruritus & & $25(83 \%)$ \\
\hline Body surface area involvement (\%) & $55[8-98]$ \\
\hline Stage of the disease & IA & $3(10 \%)$ \\
\cline { 2 - 3 } & IB & $23(76 \%)$ \\
\cline { 2 - 3 } & IIA & $4(13 \%)$ \\
\hline
\end{tabular}

Nineteen patients received NB-UVB therapy, while 11 patients received PUVA. Therapeutic and outcome data are summarized in table 2. Mean follow-up period was 36,1 \pm 13 months.

Table 2: Therapeutic and outcome data 


\begin{tabular}{|l|l|l|l|}
\hline \multicolumn{2}{|l|}{} & PUVA therapy & NB-UVB therapy \\
\hline Number of patients (\%) & $11(36 \%)$ & $19(64 \%)$ \\
\hline \multirow{2}{*}{ Skin type } & II & 1 & \\
\cline { 2 - 4 } & III & 4 & 4 \\
\cline { 2 - 4 } & IV & 5 & 11 \\
\cline { 2 - 4 } & V & 1 & 4 \\
\hline Disease stage & I A & 1 & 2 \\
\cline { 2 - 4 } & I B & 6 & 17 \\
\cline { 2 - 4 } & IIA & 4 & \\
\hline Early-stage MF & 8 & 19 \\
\hline Advanced stage MF & 3 & $1,69 \pm 0,85$ \\
\hline Average starting dose (J/cm2) & $2 \pm 0,85$ & 2,5 \\
\hline Maximal dose (J/cm2) & 6 & $55,1 \pm 16,4$ \\
\hline Cumulative dose (J/cm2) & $147 \pm 92$ & 35 \\
\hline Number of sessions to achieve complete remission (mean) & 32 & 23 \\
\hline Induction phase (mean number of sessions) & 21 & 10 \\
\hline Consolidation phase (mean number of sessions) & 11 & $16(84 \%)$ \\
\hline Complete response (n, \%) & $8(72 \%)$ & $3(16 \%)$ \\
\hline Partial response (n, \%) & $2(18 \%)$ & 8 \\
\hline Therapeutic failure (n, \%) & $1(9 \%)$ & $6(21 \%)$ \\
\hline Maintenance therapy (n) & 2 & $18 \pm 12,6$ \\
\hline Recurrence (n) & $4(36 \%)$ & $16 \pm 11,4$ & \\
\hline Recurrence free survival (months) & & \\
\hline
\end{tabular}

